\title{
Low limit of detection of the AlGaN/GaN-based sensor by the Kelvin connection detection technique
}

\author{
Hanyuan Zhang ${ }^{1}$, Ying Gan'2, Shu Yang ${ }^{1,3}$, Kuang Sheng ${ }^{1,3}$ and Ping Wang ${ }^{2}$
}

\begin{abstract}
The AlGaN/GaN-based sensor is a promising POCT (point-of-care-testing) device featuring miniaturization, low cost, and high sensitivity. BNP is an effective protein biomarker for the early diagnosis of HF (heart failure). In this work, a novel AlGaN/GaN device with the Kelvin connection structure and the corresponding detection technique was proposed. This technique can effectively suppress the background noise and improve the SNR (signal-to-noise ratio). A BNP detection experiment was carried out to verify the effectiveness of this technique. It is shown that compared with that of the traditional detection method, the LOD (limit of detection) was improved from $0.47 \mathrm{ng} / \mathrm{mL}$ to $1.29 \mathrm{pg} / \mathrm{mL}$. The BNP detection experiment was also carried out with a traditional electrochemical Au-electrode sensor with the same surface functionalization steps. The AIGaN/GaN sensor showed a better LOD than the Au-electrode sensor. Moreover, the influence of AlGaN/GaN sensor package on background noise was investigated with the mechanism of the noise source revealed. Finally, based on the optimized package, the optimal SNR quiescent operating point of the AlGaN/GaN sensor was determined. By biasing the sensor at the optimal quiescent operating point and immobilizing the magnetic beads with anti-BNP on the gate of the AlGaN/GaN sensor, the LOD for BNP detection was further improved to $0.097 \mathrm{pg} / \mathrm{mL}$.
\end{abstract}

\section{Introduction}

Heart failure (HF) is a cardiovascular disease that is one of the leading causes of death worldwide. The detection of clinically relevant cardiac biomarkers is effective in allowing the early diagnosis of HF, reducing the complications and the risk of recurrence, and ultimately reducing the economic burden on the entire medical system. Brain natriuretic peptide (BNP) is one of the most promising clinical biomarkers of HF. Based on various reports $^{1,2}$, the clinical cutoff level of BNP in chronic HF diseases is $\sim 100 \mathrm{pg} / \mathrm{mL}$. In addition, there are reports that BNP has predictive value in the diagnosis of

\footnotetext{
Correspondence: Shu Yang (eesyang@zju.edu.cn)

${ }^{1}$ College of Electrical Engineering, Zhejiang University, 310027 Hangzhou, China

${ }^{2}$ Biosensor National Special Laboratory, Department of Biomedical Engineering, Zhejiang University, 310027 Hangzhou, China Full list of author information is available at the end of the article These authors contributed equally: Hanyuan Zhang, Ying Gan
}

anthracycline-induced cardiotoxicity (AIC) ${ }^{3}$, for which the diagnostic threshold is $107.9 \mathrm{pg} / \mathrm{mL}$.

Currently, the clinical diagnostic systems used to detect protein biomarkers has drawbacks such as the need for relatively high volumes of blood samples, well-trained experts, large-scale equipment, and long processing times. A low cost, portable, and accurate POCT device is urgently needed for increased in-home monitoring of protein biomarkers for global epidemics such as HF.

The AlGaN/GaN FET sensor is a promising POCT device $^{4-6}$. With the use of different functionalization processes, the $\mathrm{AlGaN} / \mathrm{GaN}$ immunoFET ${ }^{7}$ can detect various disease biomarkers ${ }^{4,5,8-10}$. However, compared with other traditional electrochemical biosensors, such as Au electrodes, the manufacturing process for $\mathrm{AlGaN} / \mathrm{GaN}$ FET devices is more complicated, GaN epitaxy is more expensive, and the device package design is more difficult, while the advantages of $\mathrm{AlGaN} / \mathrm{GaN}$ sensors versus Au electrodes are not clear. In this work, by adopting the same surface

\section{(c) The Author(s) 2021, corrected publication 2021}

(c) (i) Open Access This article is licensed under a Creative Commons Attribution 4.0 International License, which permits use, sharing, adaptation, distribution and reproduction cc) in any medium or format, as long as you give appropriate credit to the original author(s) and the source, provide a link to the Creative Commons license, and indicate if changes were made. The images or other third party material in this article are included in the article's Creative Commons license, unless indicated otherwise in a credit line to the material. If material is not included in the article's Creative Commons license and your intended use is not permitted by statutory regulation or exceeds the permitted use, you will need to obtain permission directly from the copyright holder. To view a copy of this license, visit http://creativecommons.org/licenses/by/4.0/. 
functionalization method for the traditional Au-electrode electrochemical sensor and the AlGaN/GaN FET sensor (Device A in this work), the BNP detection performances of the two sensors were compared.

The two-dimensional electron gas (2DEG) structure formed by the AlGaN/GaN heterojunction can achieve both high carrier mobility (typically, $2000 \mathrm{~cm}^{2} / \mathrm{V} \cdot \mathrm{s}$ ) and high electron density (typically, $\left.\sim 1 \times 10^{13} \mathrm{~cm}^{-2}\right)^{11}$; thus, the current sensitivity $S_{\mathrm{I}}$, which represents the ability of the $\mathrm{AlGaN} / \mathrm{GaN}$ device to amplify the biosignal, is very high $^{12}$. Therefore, methods to improve the sensitivity of AlGaN/GaN sensors have been extensively studied ${ }^{11-19}$. However, in practical applications, the background noise of the device may also be amplified, and the benefits of the high sensitivity will then be offset. As a result, it is very important to determine how to reduce the background noise of the device to improve the overall performance. To reduce the background noise and improve the SNR of the device, we proposed using a Kelvin connection device structure and the corresponding test method. The fourprobe test method is an electrical measurement method used to accurately measure resistance while excluding the influence of the series resistance. The principle involves decoupling the current loop and the voltage measurement loop so that the current and voltage signals can be measured accurately and the resistance can be calculated accurately. The Kelvin connection technique was also used in traditional ISFET sensors ${ }^{20}$. However, the role of the Kelvin connection in ISFETs and AlGaN/GaN devices is different. In the ISFET device, the Kelvin connection technique is used to isolate the terminal voltage measurement loop from the current source loop. However, in the $\mathrm{AlGaN} / \mathrm{GaN}$ sensor, it is used to suppress the background noise and improve the SNR. The difference in the Kelvin connection technique of the two devices originates from the differences in sensor structures and sensor characteristics. In the fabrication process of the CMOScompatible ISFET, it is relatively difficult to produce a successful passivation layer to selectively expose the active sensing area and to protect the metal leads from the solution at the same time. Therefore, there are long source and drain regions that are highly doped with $\mathrm{Si}$, and these are simplify the use of materials such as thick epoxy to cover the metal leads ${ }^{21}$. The highly doped drift regions provide excessive series resistance, which reduces the current sensitivity of the FET sensor ${ }^{11}$. Moreover, since the $\mathrm{SiO}_{2}$ gate oxide layer grown by the thermal oxidation process is easily penetrated by the ions in the solution, which causes the turn-on voltage shift and device failure, the poly gate is connected to the thick $\mathrm{SiN}_{\mathrm{x}}$ layer acting as the passivation layer and the gate-sensitive membrane ${ }^{21}$. The $\mathrm{SiN}_{\mathrm{x}}$ layer provides excessive series capacitance to the gate of the ISFET, and the current sensitivity is further reduced. Therefore, to compensate for the poor current sensitivity, source and drain follower read-out circuits are often used in sensor arrays to ensure that the reference electrode is grounded ${ }^{22}$. In the ISFET read-out circuit, both drain-to-source voltage $\left(V_{\mathrm{DS}}\right)$ and drain-to-source current $\left(I_{\mathrm{DS}}\right)$ are constant, and the change in the solution will cause a change in the gate voltage $\Delta V_{\mathrm{G}}$ through the double layer on the solution $/ \mathrm{SiN}_{\mathrm{x}}$ interface. $\Delta V_{\mathrm{G}}$ is equal to the output signal source voltage $\left(V_{\mathrm{S}}\right)$ and is amplified by the off-chip amplifier. To accurately read the $V_{\mathrm{S}}$, the voltage readout circuit loop is decoupled with the current-flowing circuit loop based on the Kelvin connection technique. In the source and drain follower read-out circuit, the $\Delta V_{\mathrm{G}}$ signal is not amplified, and the signal-to-noise ratio (SNR) is limited by the electronic noise of the read-out circuit instead of the ISFET device itself. In this work, the AlGaN/GaN sensor is biased with a constant $V_{\mathrm{DS}}$ voltage, and $I_{\mathrm{DS}}=\Delta V_{\mathrm{G}} \times g_{\mathrm{m}}$ is read out as the output signal (equivalent to $R_{\mathrm{DS}}=V_{\mathrm{DS}} / I_{\mathrm{DS}}$ when $V_{\mathrm{DS}}$ is a constant). $\Delta V_{\mathrm{G}}$ is amplified by $g_{\mathrm{m}}$, and the SNR is limited by both $g_{\mathrm{m}}$ and the noise of the AlGaN/GaN sensor itself. The reason for using the Kelvin connection technique in the $\mathrm{AlGaN} / \mathrm{GaN}$ sensor is that according to our previous study ${ }^{11}$, the series resistance reduces the $g_{\mathrm{m}}$ of the sensor. The Kelvin connection technique can reduce the influence of the series resistance and improve $g_{\mathrm{m}}$. However, in the experiment, we unexpectedly found that the Kelvin connection technique also contributes a positive role in the noise performance of the device; it not only reduces the thermal white noise on the series resistance but also effectively reduces the overall $1 / \mathrm{f}$ mode background noise of the $\mathrm{AlGaN} / \mathrm{GaN}$ sensor and improves the LOD.

Passivation is one of the key challenges in liquid sensors $^{7}$. It is well known that the quality of the package will affect the device lifetime ${ }^{23-27}$ and the safe operating area $(\mathrm{SOA})^{28}$. However, the influence of the package on the device background noise has not been discussed. PECVD-grown $\mathrm{SiO}_{2}$ and $\mathrm{SiN}_{\mathrm{x}}$ are CMOS-compatible packaging materials often used as liquid sensors ${ }^{17,18,29,30}$. In our previous work, the $\mathrm{PI} / \mathrm{SiN}_{\mathrm{x}} / \mathrm{SiO}_{2}$ multilayer package was found to effectively suppress the leakage current $I_{\mathrm{G}}$ flowing between the metal and the reference electrode in the solution under different voltage stresses $^{31}$. By comparing the correlation between $I_{\mathrm{G}}$ and the background noise for the $\mathrm{PI} / \mathrm{SiN}_{\mathrm{x}} / \mathrm{SiO}_{2}$ multilayer package and the traditional $\mathrm{SiO}_{2} / \mathrm{SiN}_{\mathrm{x}}$ package, we found that the package material affects the device background noise by influencing the gate leakage current. The mechanism of the process was also illustrated by the experimental results.

The quiescent operating point of the AlGaN/GaN sensor is an important factor that influences the sensitivity ${ }^{32}$. However, the effect of the quiescent operating point on the SNR has not been investigated. In this work, the 
SNR of the AlGaN/GaN device with different quiescent operating points was determined. Another method to detect BNP was developed by immobilizing anti-BNP on the magnetic beads and adsorbing the magnetic beads on the surface of the $\mathrm{AlGaN} / \mathrm{GaN}$ open gate area through a magnet ${ }^{8}$. The device (Device B in this work) was biased at the optimal quiescent operating point, and the LOD for BNP detection was further improved to $0.097 \mathrm{pg} / \mathrm{mL}$.

\section{Results}

\section{BNP detection: Au-electrode with EIS}

Figure 1a shows the change in the charge transfer resistance $\left(R_{\mathrm{CT}}\right)$ of the Au electrode with the functionalization process in the Electrochemical Impedance Spectroscopy (EIS) test. The $R_{\mathrm{CT}}$ increased from 1 to 2 after the $\mathrm{Au}$ electrode was coated with BNP antibody (anti-BNP) and further increased from 2 to 3 after the binding sites were blocked by bovine albumin (BSA). When BNP and antiBNP were specifically bound, $R_{\mathrm{CT}}$ decreased from 3 to 4 , and the amount of $R_{\mathrm{CT}}$ decrease was $\Delta R_{\mathrm{CT}}$. The inset shows the circuit model used to simulate the $R_{\mathrm{CT}}$ value in the EIS curve. $R_{\mathrm{S}}$ represents the series resistance, including the solution resistance and the series resistance in the circuit. $R_{\mathrm{CT}}$ is the charge transfer resistance, and constant phase element (CPE) is used to simulate the capacitance of the double layer and compensate for the nonhomogeneity in the system. Figure $1 \mathrm{~b}$ shows the plot of $\Delta R_{\mathrm{CT}}$ versus different BNP concentrations ranging from $1 \mathrm{ppb}$ to $500 \mathrm{ppb} . R_{\mathrm{CT} 3}$ is the charge transfer resistance of the $\mathrm{Au}$ electrode after BSA blocking, and the standard deviation is $\sigma_{R C \mathrm{~T} 3}=849.4 \Omega$, which can be regarded as the background noise. Substituting $\sigma_{\mathrm{RCT} 3}$ three times into $\mathrm{Y}$ of the linear fitting line of $\Delta R_{\mathrm{CT}}-\mathrm{BNP}$, the $\mathrm{X}$ value obtained is the LOD of BNP detection by the $\mathrm{Au}$ electrode. In this work, the LOD of the Au-electrode is $2.73 \mathrm{ppb}$.
The selectivity of the $\mathrm{Au}$ electrode treated with this functionalization method was determined, and the results are shown in Fig. S1 in the supplementary material.

\section{Fabrication of the AlGaN/GaN sensor}

Two types of $\mathrm{AlGaN} / \mathrm{GaN}$ devices designed in this work, Device A and Device B, are shown in Fig. 2b, c. The fabrication and packaging process of Device $B$ has been described $^{31}$, and the only difference between Device A and Device $B$ is that the open gate area was deposited with a layer of $\mathrm{Au}$ as the sensitive membrane. The $\mathrm{PI} / \mathrm{SiN}_{\mathrm{x}} /$ $\mathrm{SiO}_{2}$ multilayer was used as the package in this work. There are four terminals for both Device A and Device B. High force (H. F) and high sense (H. S) terminals are connected to the drain, and low force (L. F) and low sense (L. S) terminals are connected to the source, and the circuit schematic and the actual device connection diagram are shown in Fig. 2a.

\section{BNP detection: AIGaN/GaN sensor (Device A) with Au gate}

Figure $3 \mathrm{a}$ shows the change in $I_{\mathrm{DS}}$ output with the functionalization process of Device A. The trend of $I_{\mathrm{DS}}$ change was similar to that of the Au electrode; after the $\mathrm{Au}$ gate was coated with anti-BNP, $I_{\mathrm{DS}}$ was reduced, and in other words, the channel resistance of the AlGaN/GaN device increased. Then, the Au gate was blocked by BSA, and the $I_{\mathrm{DS}}$ increased. Finally, after BNP and anti-BNP were specifically bonded, the $I_{\mathrm{DS}}$ decreased again.

Figure $3 \mathrm{~b}$ shows the $R_{\mathrm{DS}}$ baseline measurement for the Kelvin connection technique and two-probe test method after the BSA blocking process. $V_{\mathrm{DS}}$ was fixed to be $0.4 \mathrm{~V}$. It is shown that $R_{\mathrm{DS}-4 \text { probe }}$ (drain-to-source resistance measured by Kelvin connection technique) was smaller than $R_{\mathrm{DS}-2 \text { probe }}$ (drain-to-source resistance measured by two-probe method) by $\sim 5 \Omega$ in absolute value, and its
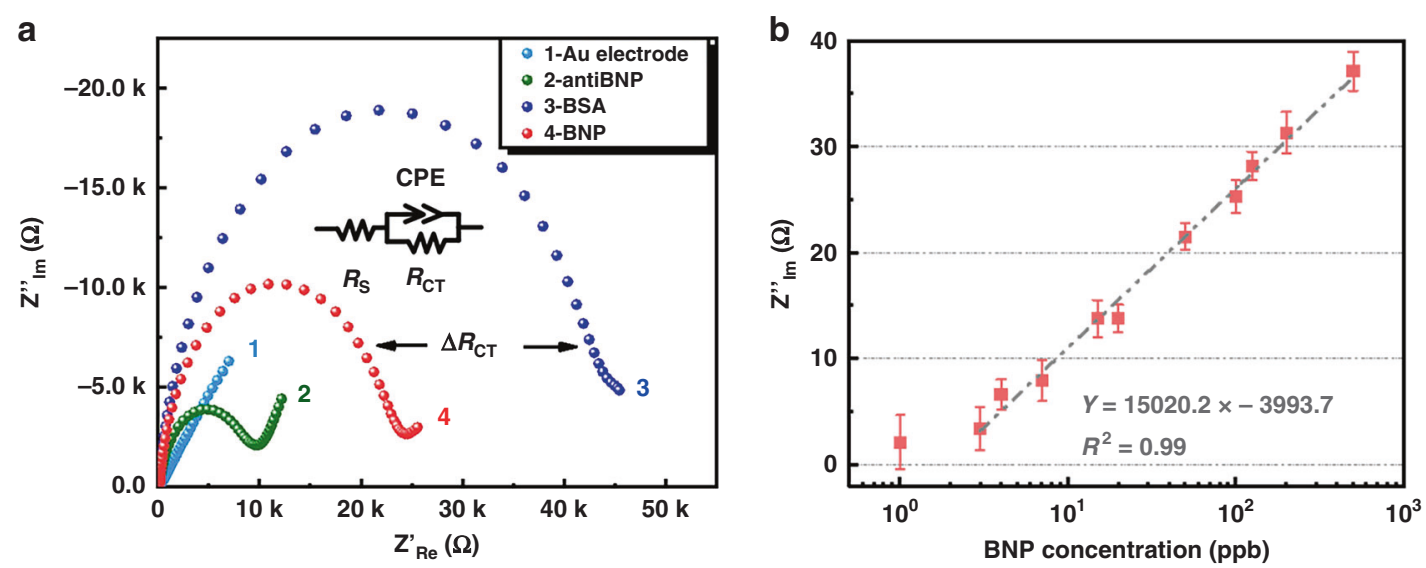

Fig. 1 BNP detection results based on Au-electrode. a Change of $R_{C T}$ in the EIS with the surface functionalization process. The inset is the circuit model used to simulate the $R_{C T}$ value. $Z^{\prime}{ }_{R e}$ is the real part, and $Z^{\prime}{ }_{\mathrm{Im}}$ is the imaginary part of the impedance in the EIS of the Au electrode. $\mathbf{b}$ Change in $R_{C T}$ at different concentrations of BNP. 

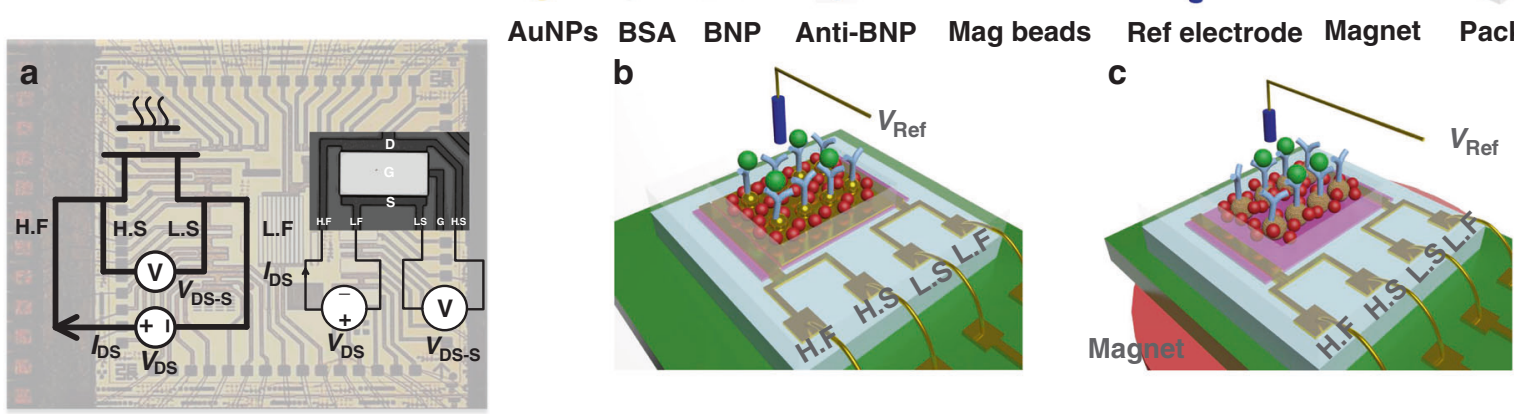

Fig. 2 Schematic diagram and structure model diagrams of the Kelvin connection devices. a Diagram of the Kelvin connection device; high force (H. F) and high sense (H.S) are connected to the drain terminal, low force (L. F) and low sense (L. S) are connected to the source terminal. $V_{D S}$ voltage is applied between the H. F and L. F to form the current loop. The voltage between the H. S and L. S is measured as $V_{D S-S}$. The diagram on the left is the schematic diagram and that on the right is the OM (Optical microscope (OM) top view of the real device. $\mathbf{b}$ Device $A$. The open gate area of device A is deposited by the Au layer. AuNPs (Au nanoparticles) are electroplated on the Au gate, and the anti-BNP is immobilized on the AuNPs to selectively detect BNP. c Device B. The open gate area of Device B is bare AlGaN. Magnetic beads immobilized with anti-BNP are absorbed on the AlGaN surface with a permanent magnet on the back side of the chip.

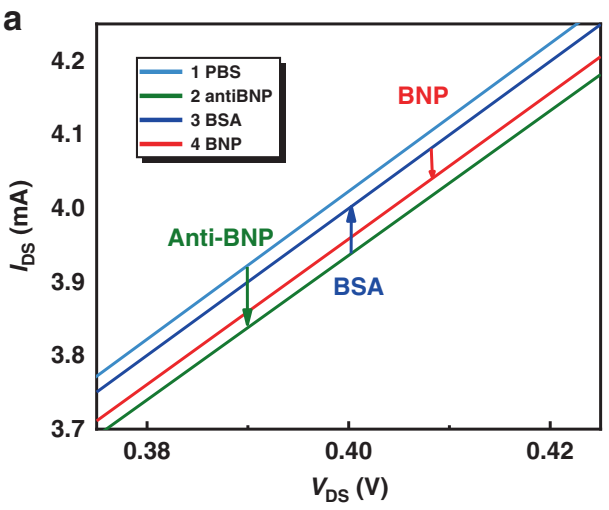

C

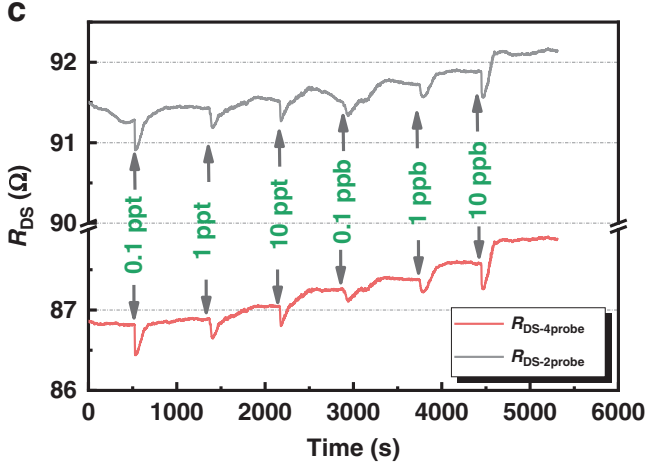

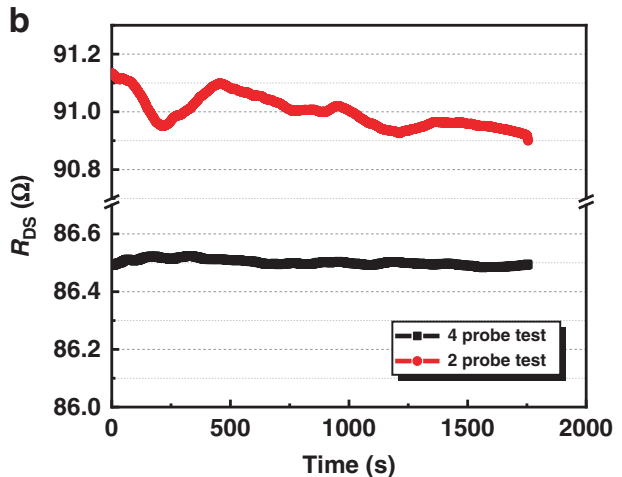

d

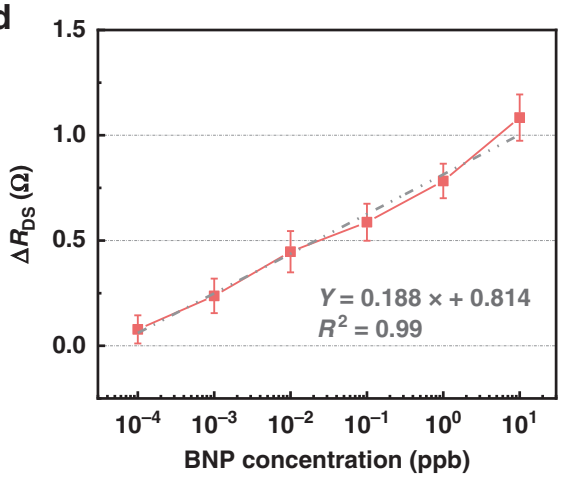

Fig. 3 BNP detection results based on AIGaN/GaN sensor with Au gate membrane (Device A). a Change of IDS output of Device A with the surface functionalization process. $\mathbf{b} R_{\mathrm{DS}}$-t baseline comparison $\left(V_{\mathrm{DS}}=0.4 \mathrm{~V}\right)$ between the Kelvin connection test and the traditional two-probe test. c $R_{\mathrm{DS}-4 \text { probe }} \mathrm{t}$ and $R_{\mathrm{DS}-2 \text { probe }}$-t response to BNP with concentrations ranging from $0.1 \mathrm{ppt}$ to $10 \mathrm{ppb}$ using Device $\mathrm{A}$ as the transducer and anti-BNP immobilized on the Au gate. $\mathbf{d} \triangle R_{\mathrm{DS}}$ results of BNP detection.

variation was also smaller than that of $R_{\mathrm{DS}-2 \text { probe }}$ The absolute value of $R_{\mathrm{DS}-4 \text { probe }}$ was reduced because it eliminated the influence of the series resistance in both the measurement circuit and the thin film resistance of the wafer. The reduction in the resistance variation indicates that the series resistance can not simply be regarded as a constant resistance; it also introduced noise into BNP measurements, thereby affecting the LOD of the sensor. 
The standard deviation of the Kelvin connection technique $\sigma_{R \mathrm{DS}}$-4probe was $0.09 \Omega$, while that of the two-probe method $\sigma_{R \mathrm{DS}-2 \text { probe }}$ was $0.251 \Omega$.

Figure 3c shows the $R_{\mathrm{DS}-4 \text { probe }}$ and $R_{\mathrm{DS}-2 \text { probe }}$ responses to $\mathrm{BNP}$ at concentrations ranging from $0.1 \mathrm{ppt}$ to $10 \mathrm{ppb}$, indicating that $R_{\mathrm{DS}-4 \text { probe }}$ showed a more stable response than $R_{\mathrm{DS}-2 \text { probe. }}$ Figure $3 \mathrm{~d}$ shows the plot of $\Delta R_{\mathrm{DS}}$ (drainto-source resistance change) versus different concentrations of BNP. Substituting $3 \sigma_{R \mathrm{DS}}-4$ probe into the linear fitting curve of Fig. 3d, the LOD of Device A using the Kelvin connection technique is 1.29 ppt. On the other hand, the LOD of the two-probe testing method is only $0.47 \mathrm{ppb}$ due to the larger background noise.

The selectivity of Device A with this functionalization method was determined, and the results are shown in Fig. S2 in the supplementary material.

\section{Package and background noise}

Packaging materials need to ensure that the device can be safely operated in solution. An important indicator for characterizing packaging quality is the gate leakage current through the reference electrode of the device under different $V_{\mathrm{G}}$ bias voltages. This work showed that the gate leakage current affects the background noise in the $R_{\mathrm{DS}}$ baseline of the device. Therefore, optimizing the quality of the package not only directly expands the safe operating area of the device but also effectively reduces the background noise and enables the sensor to obtain a lower LOD. Figure $4 \mathrm{a}-\mathrm{e}$ are the baseline test results of Device B with different $V_{\mathrm{G}}$ biases ranging from $0 \mathrm{~V}$ to $-3.8 \mathrm{~V}$. The packaging material was $\mathrm{PI} / \mathrm{SiN}_{\mathrm{x}} / \mathrm{SiO}_{2}$, which was demonstrated to effectively suppress the gate leakage current in prior work ${ }^{31}$. Figure $4 \mathrm{f}$ shows the baseline test of Device B with $V_{\mathrm{G}}=0 \mathrm{~V}$, but the packaging material was traditional $\mathrm{SiN}_{\mathrm{x}} / \mathrm{SiO}_{2}$. The leakage $I_{\mathrm{G}}$ current was $<10^{-9}$ A for the $\mathrm{PI} / \mathrm{SiN}_{\mathrm{x}} / \mathrm{SiO}_{2}$ package, while $I_{\mathrm{G}}$ was on the order of $10^{-6} \mathrm{~A}$ for the $\mathrm{SiN}_{\mathrm{x}} / \mathrm{SiO}_{2}$ package. Moreover, it is obvious from Fig. 4f that $I_{\mathrm{G}}$ and $R_{\mathrm{DS}}$ showed a strong correlation.

\section{BNP detection: AIGaN/GaN sensor (Device B) with magnetic beads}

The $I_{\mathrm{DS}}$ background noise $N_{\mathrm{IDS}}$ is obtained by calculating the standard deviation of the steady-state $I_{\mathrm{DS}}$ $(500 \mathrm{~ms} / \mathrm{sample}$, sample time $2000 \mathrm{~s})$ with $V_{\mathrm{DS}}=0.4 \mathrm{~V}$. The $I_{\mathrm{DS}}$ response $\Delta I_{\mathrm{DS}}$ is obtained from the change in surface potential $\psi_{0}$ multiplied by the transconductance $g_{\mathrm{m}}$. Thus, the SNR is defined as:

$$
\mathrm{SNR}=\frac{\Delta I_{\mathrm{DS}}}{N_{\mathrm{IDS}}}=\psi_{0} \frac{g_{\mathrm{m}}\left(V_{\mathrm{G}}\right)}{N_{\mathrm{IDS}}}
$$

Since $\psi_{0}$ depends only on the interaction between the solution and the sensitive membrane, so $g_{\mathrm{m}} / N_{\mathrm{IDS}}$ represents the SNR of the device per voltage change in surface potential. Figure $5 \mathrm{c}$ shows the test results of $g_{\mathrm{m}}$ of Device $\mathrm{B}$ and its $g_{\mathrm{m}} / N_{\mathrm{IDS}}$ under different $V_{\mathrm{G}}$ biases. The SNR of Device $B$ reached the optimal value at $V_{\mathrm{G}}=-3.8 \mathrm{~V}$.

The reason that Device A could not be operated with a negative $V_{\mathrm{G}}$ bias is that the SOA (safe operating area) of Device A is very small, so the Au metal on the gate region would be corroded ${ }^{28}$ at a negative $V_{\mathrm{G}}$ bias. To bias the device at the optimal SNR quiescent operating point, Device B (with a larger SOA) was used as the transducer. To immobilize the anti-BNP on the gate-sensitive area, anti-BNP was first immobilized on magnetic microbeads and then the microbeads were adsorbed on the open gate area of Device B with a magnet. When BNP specifically bonded with anti-BNP, it caused a potential change $\Delta V_{\mathrm{G}}$ on the gate of Device $\mathrm{B}$ so that the channel resistance $R_{\mathrm{DS}}$ was read out as the output signal. The advantage of immobilizing the anti-BNP on the magnetic microbeads is that this method for detecting BNP can be used on Device B, which has a larger SOA. Moreover, the expensive AlGaN/GaN sensor can be easily refreshed and reused by removing the magnet and washing away the microbeads from the gate area. The BNP detection results are shown in Fig. 5a, b. The background noise of Device B at $V_{\mathrm{G}}=-3.8 \mathrm{~V}$ was $0.18 \Omega$, and the calculated LOD was $0.097 \mathrm{ppt}$.

The selectivity of Device B with this functionalization method was determined, and the results are shown in Fig. S3 in the supplementary material.

\section{Discussion}

The Spearman correlation coefficients of the $I_{\mathrm{G}}$ and $R_{\mathrm{DS}}$ (Fig. 4) are compared in Fig. 6a. This result shows that for the $\mathrm{PI} / \mathrm{SiN}_{\mathrm{x}} / \mathrm{SiO}_{2}$ package, the gate leakage current $I_{\mathrm{G}}$ and the baseline noise $R_{\mathrm{DS}}$ presented a weak negative correlation between -0.62 and 0 with any quiescent working points of $V_{\mathrm{G}}$ from $0 \mathrm{~V}$ to $-3.8 \mathrm{~V}$. However, for the $\mathrm{SiN}_{\mathrm{x}} /$ $\mathrm{SiO}_{2}$ package, the gate leakage current $I_{\mathrm{G}}$ had a strong positive correlation of 0.83 with the baseline noise $R_{\mathrm{DS}}$ with $V_{\mathrm{G}}=0 \mathrm{~V}$.

To illustrate the mechanism causing this difference in the correlations of two types of package materials, the steady-state values of $I_{\mathrm{G}}$, excluding the capacitance effect under different $V_{\mathrm{G}}$ voltage stresses, are plotted in Fig. 6b. For the $\mathrm{PI} / \mathrm{SiN}_{\mathrm{x}} / \mathrm{SiO}_{2}$ package, the $I_{\mathrm{G}}-V_{\mathrm{G}}$ curve obeyed Ohm's Law:

$$
J=e n \mu E=e n \mu V / a
$$

where $e$ is the charge of an electron, $n$ is the intrinsic carrier density, $\mu$ is the carrier mobility, $E$ is the electric field of the current, $V$ is the voltage, and $a$ is the thickness of the material. This indicated that the high-resistance 
a

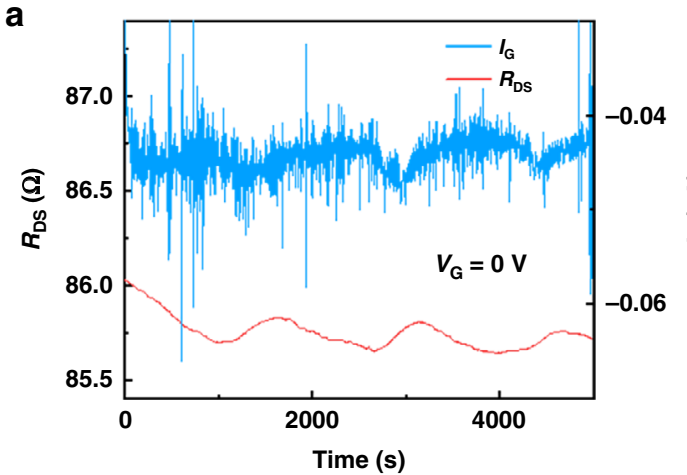

C
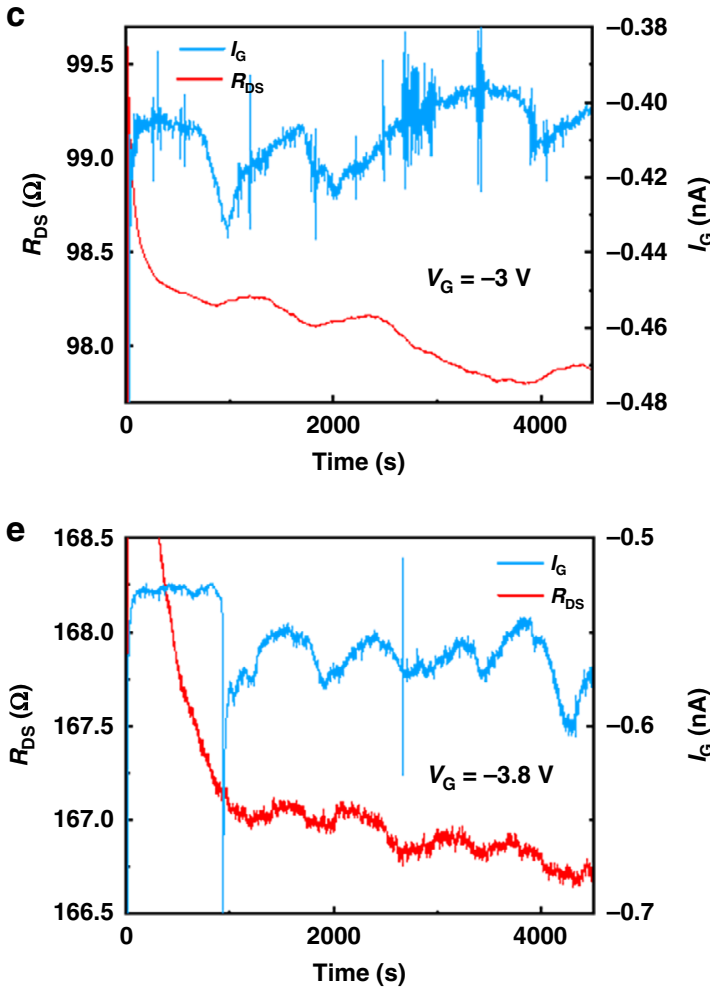

b

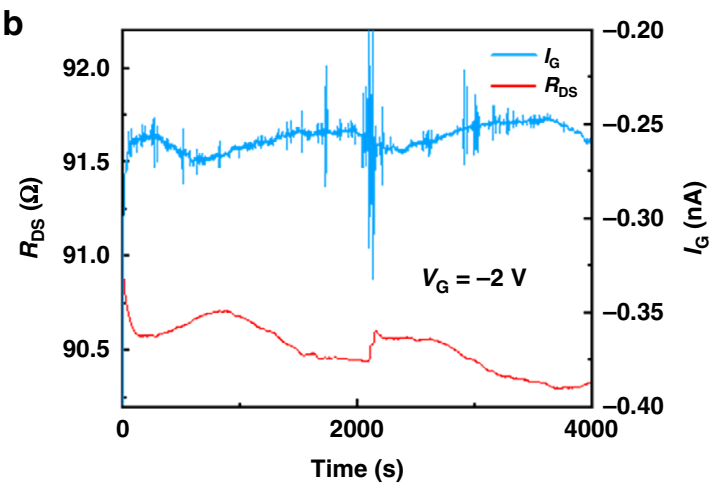

d

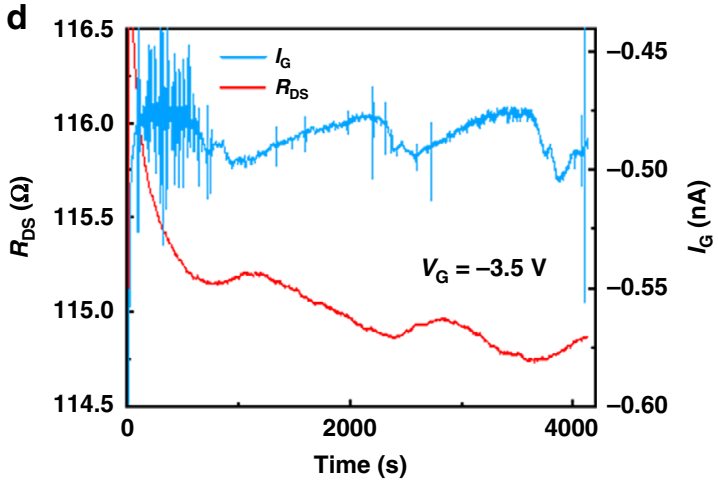

$\mathbf{f}$

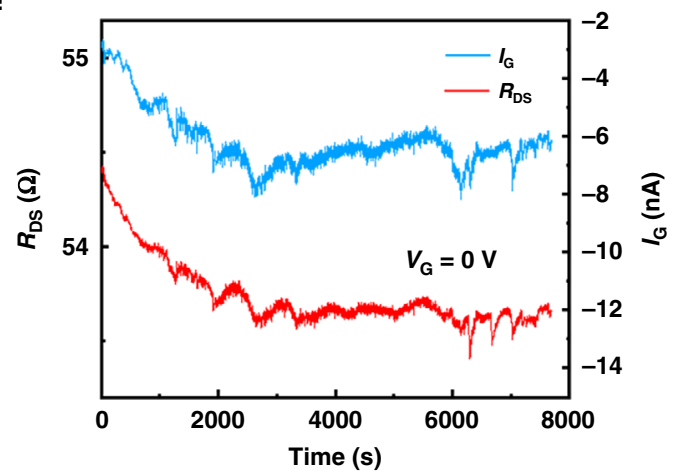

Fig. 4 The experimental results of the gate leakage current $I_{\mathrm{G}}$ and $R_{\mathrm{DS}}$ background noise of the AIGaN/GaN sensors. a-e are the baseline tests of Device $B$ with different $V_{G}$ biases; the packaging material is $\mathrm{PI} / \mathrm{SiN}_{\mathrm{x}} / \mathrm{SiO}_{2}$. $R_{\mathrm{DS}}$ is the Kelvin connection test resuts and $I_{\mathrm{G}}$ is the gate leakage current through the reference electrode. $R_{D S}$ and $I_{G}$ were measured simultaneously with $\mathbf{a} V_{G}=0 \mathrm{~V}, \mathbf{b} V_{G}=-2 \mathrm{~V}, \mathbf{c} V_{G}=-3 \mathrm{~V}, \mathbf{d} V_{G}=-3.5 \mathrm{~V}$, e $V_{G}=-3.8 \mathrm{~V}$. $\mathbf{f}$ Baseline test of Device $\mathrm{B}$ with $V_{\mathrm{G}}=0 \mathrm{~V}$; the packaging material is $\mathrm{SiN}_{\mathrm{x}} / \mathrm{SiO}_{2}$.

package layer completely blocked the contact between the metal and the solution, and most of the potential between the source/drain metals and the gate reference electrode fell on the package layer. The gate leakage current was dominated by the package layer featuring ohmic characteristics; this finding indicated that there were no external carriers injected into the package layer, and only the internal carriers were driven by the $V_{\mathrm{G}}$ voltage stress to form the leakage current $I_{\mathrm{G}}$. At this time, the leakage current level was not strong enough to cause a significant voltage fluctuation at the open gate area of the device; thus, the $R_{\mathrm{DS}}$ baseline was not strongly influenced by the $I_{\mathrm{G}}$, so the correlation between $I_{\mathrm{G}}$ and the background baseline $R_{\mathrm{DS}}$ was weak.

For the $\mathrm{SiN}_{\mathrm{x}} / \mathrm{SiO}_{2}$ package, the $I_{\mathrm{G}}-V_{\mathrm{G}}$ curve obeyed the Butler-Volmer Law:

$$
J=J_{0}\left[\exp \left(-\frac{\alpha n F \eta}{R T}\right)-\exp \left(\frac{\beta n F \eta}{R T}\right)\right]
$$

where $J_{0}$ is the exchange current density, $R$ is the ideal gas constant, $T$ is the absolute temperature, $n$ is the number 

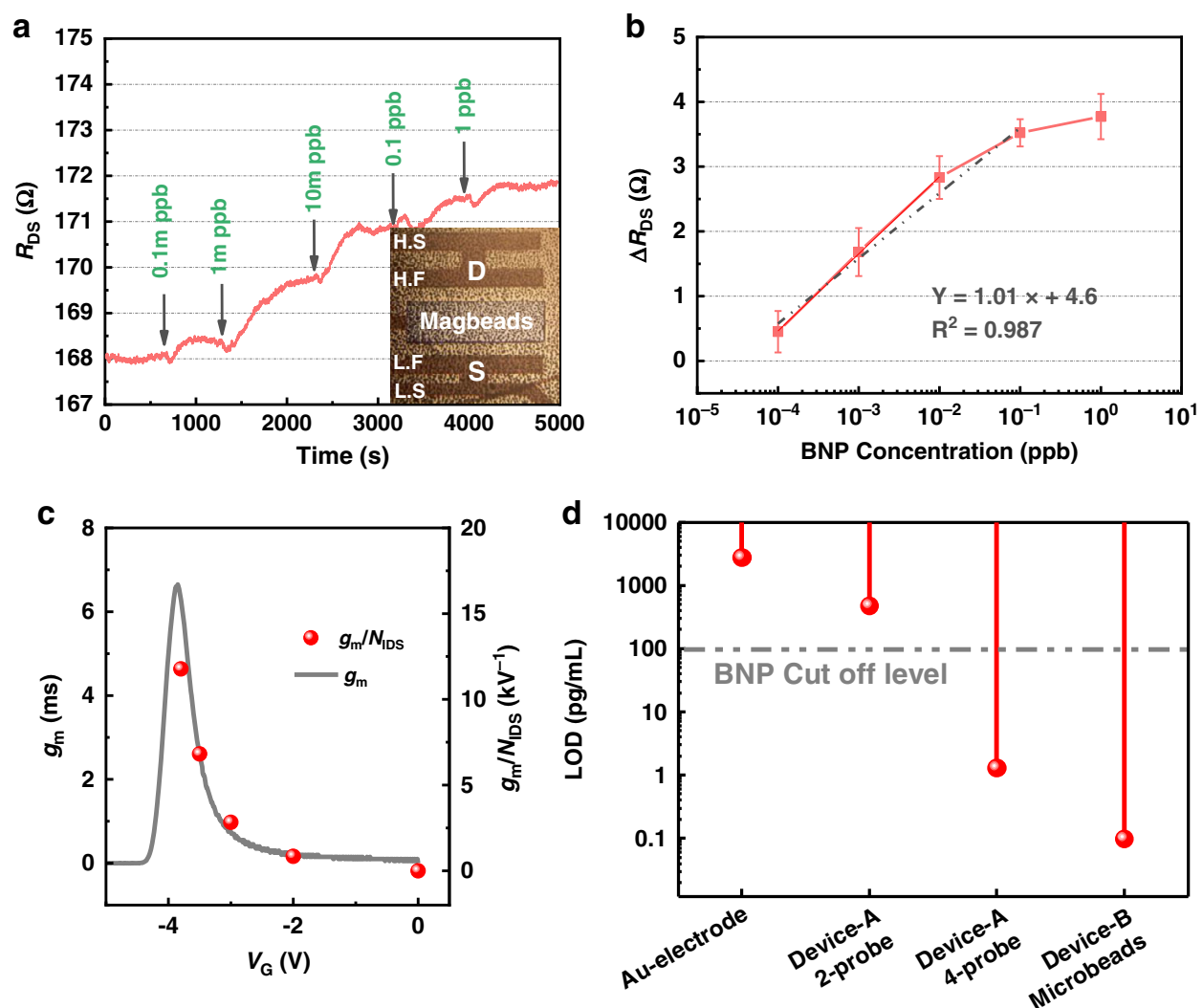

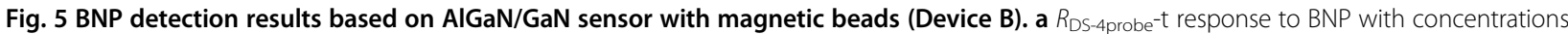
ranging from $0.1 \mathrm{ppt}$ to $1 \mathrm{ppb}$, using Device $B$ as the transducer and anti-BNP immobilized on the magnetic beads. The inset is the optical microscope $(\mathrm{OM})$ top view of the AlGaN/GaN device immobilized with magnetic beads. $\mathbf{b} \Delta R_{\mathrm{DS}-4 \text { probe }}$ results of BNP detection with the Kelvin connection technique and $V_{G}=-3.8 \mathrm{~V}$. c Transconductance of Device $B$ and its SNR versus different $V_{G}$ biases of the reference electrode. d Comparison of the LOD values for the Au-electrode, Device A using the two-probe method, Device A using the Kelvin connection technique, and Device $B$ biased at the optimal SNR quiescent working point $\left(V_{G}=-3.8 \mathrm{~V}\right.$, using the Kelvin connection technique.
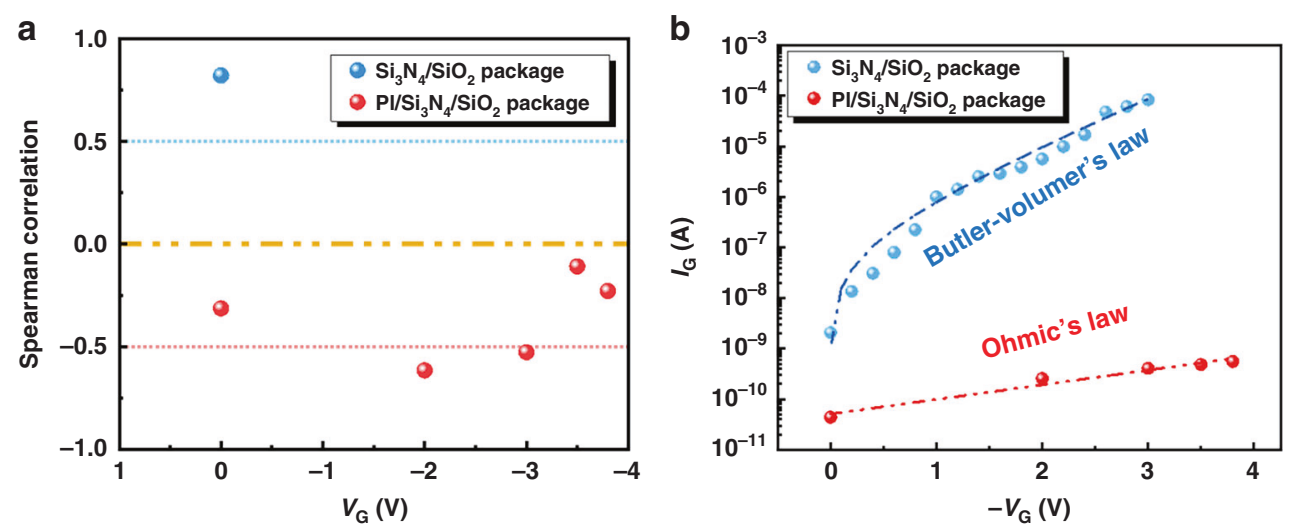

Fig. 6 Influence of the packaging material to the background noies. a Spearman correlation of $I_{G}$ and $R_{D S}$ in Fig. 4. b Steady-state value of $I_{G}$ with different $V_{\mathrm{G}}$ biases and different packages.

of electrons participating in the reaction, $F$ is the Faraday constant, $\alpha$ is the transfer coefficient of the oxidation reaction, $\beta$ is the transfer coefficient of the reduction reaction, and $\eta$ is the overpotential of the electrochemical reaction. This indicated that the $\mathrm{SiN}_{\mathrm{x}} / \mathrm{SiO}_{2}$ package failed to thoroughly protect the source/drain metals from the solution. There were local areas of metal in direct contact with the solution. At this time, the $V_{\mathrm{G}}$ mainly fell on the 
interface between the metal and the solution, which led to the overpotential $\eta$. The electrochemical reaction was driven by $\eta$, and the rate of the electrochemical reaction became the dominant factor in gate leakage current $I_{\mathrm{G}}$ at this time. The electrochemical reaction rate was also related to the surface state at the interface, the concentrations of reactants, the convection velocity of the solution, and other factors, so there were large fluctuations in $I_{\mathrm{G}}$. Although only a small part of the metal was in contact with the solution, the $I_{\mathrm{G}}$ was much larger with this packaging system than that in the $\mathrm{PI} / \mathrm{SiN}_{\mathrm{x}} / \mathrm{SiO}_{2}$ package. This fluctuating leakage current $I_{\mathrm{G}}$ flowed through the solution resistance and formed a fluctuating voltage drop on the open gate area of the device, which was further reflected in the device baseline as the $R_{\mathrm{DS}}$ fluctuation, in other words, as the background noise. When $I_{\mathrm{G}}$ decreased (the absolute value increased), the open gate voltage of the device increased, so the channel resistance $R_{\mathrm{DS}}$ decreased. As a result, we found that there was a strong positive correlation between $I_{\mathrm{G}}$ and $R_{\mathrm{DS}}$ for the $\mathrm{SiN}_{\mathrm{x}} / \mathrm{SiO}_{2}$ package.

Consequently, the quality of the packaging material affects the leakage current on the reference electrode and then affects the background noise of the device. If the packaging material is perfect and the leakage current on the reference electrode obeys Ohm's law, then $I_{\mathrm{G}}$ and $R_{\mathrm{DS}}$ show weak correlations, and other effects dominate the background noise. On the other hand, if the packaging material is poor, a local area of the metal electrode is in contact with the solution, and $I_{\mathrm{G}}-V_{\mathrm{G}}$ obeys the Butler-Volmer Law. Then, $I_{\mathrm{G}}$ and $R_{\mathrm{DS}}$ show a strong positive correlation, and the leakage current dominates the background noise. Therefore, high-quality packaging not only increases the lifetime of the device and enlarges the safe operating area but also reduces the background noise of the device, thereby increasing its LOD.

In summary, the LODs for BNP detection by the $\mathrm{Au}$ electrode, Device A using the two-probe method, Device A using the Kelvin connection technique, and Device B using magnetic beads as the sensitive membrane are compared in Fig. 5d. Compared with the Au electrode, the AlGaN/GaN FET device, which was made with exactly the same functionalization process, improved the BNP LOD from $2.73 \mathrm{ppb}$ to $0.47 \mathrm{ppb}$. The Kelvin connection technique designed in this work was proven to effectively reduce the background noise and increase the LOD to $1.29 \mathrm{ppt}$, which was lower than the cutoff concentration for HF disease. This method can be used not only in the AlGaN/GaN device but also in any other kind of FET sensor. The quality of the package was very important to the AlGaN/GaN sensor, since it can affect the magnitude of the leakage current to the reference electrode and thereby influence background noise. The optimal SNR was also shown in this work. It was found that as long as the package effectively protected the metal from undergoing electrochemically reactions with the solution, the optimal SNR quiescent operating point roughly coincided with the peak $g_{\mathrm{m}}$ point. Biasing the device at the optimal SNR working point led to an extremely low LOD of 0.097 ppt. Table 1 compares the LODs exhibited by various immunosensors for various proteins. Table 1 also shows comparisons of the LOD for BNP detection in this work (Device B) with LODs of other sensors used to detect BNP, AlGaN/GaN sensors used to detect other proteins with a similar method (antigen antibody specific binding) and other two-dimensional material devices and nanodevices. Compared with the electrochemical sensors described in references $^{33,34}$, the LOD is this work is more than 40 times smaller. Compared with other $\mathrm{AlGaN} / \mathrm{GaN}$ immunosensors in references ${ }^{35-40}$, the LOD is more than 3 orders of magnitude lower. Compared with the graphene sensor and the silicon nanowire in references ${ }^{36,37}$, the LOD is approximately 4 orders of magnitude lower.

\section{Materials and methods \\ Surface functionalization and BNP detection}

For the $\mathrm{Au}$ electrode, the first step was to perform surface activation in a $0.1 \mathrm{M} \mathrm{H}_{2} \mathrm{SO}_{4}$ solution with a cyclic

Table 1 Comparison of LODs for detection of different proteins by different transducer platforms

\begin{tabular}{|c|c|c|c|c|}
\hline Transducer & Bioprobe & Target molecule & LOD & Refs. \\
\hline AlGaN/GaN & Anti-BNP on microbeads & BNP & $97 \mathrm{fg} / \mathrm{mL}$ & This work \\
\hline Screen-printed carbon electrodes & Peroxidase-labeled BNP antibodies on gold nanoparticles & BNP & $4 \mathrm{pg} / \mathrm{mL}$ & 33 \\
\hline Silver disk electrode & Acetylcholinesterase-labeled anti-BNP antibodies & BNP & $10 \mathrm{ng} / \mathrm{mL}$ & 34 \\
\hline AlGaN/GaN HEMT & NT-proBNP specific aptamer & NT-proBNP & $0.22 \mathrm{ng} / \mathrm{mL}$ & 35 \\
\hline AlGaN/GaN HEMT & FHC antibody & Protein-peptide & $56.7 \mathrm{ng} / \mathrm{mL}$ & 38 \\
\hline AIGaN/GaN HEMT & Botulinum antibody & Botulinum toxin & $1 \mathrm{ng} / \mathrm{mL}$ & 39 \\
\hline AlGaN/GaN HEMT & Anti-NT-proBNP & NT-proBNP & $181 \mathrm{pg} / \mathrm{mL}$ & 40 \\
\hline Graphene & Human anti-EGP & $\mathrm{EGP}$ & $1 \mathrm{ng} / \mathrm{mL}$ & 36 \\
\hline Silicon nanowire & Anti-APOA1 & hAPOA1 & $1 \mathrm{ng} / \mathrm{mL}$ & 37 \\
\hline
\end{tabular}


voltammetry (CV) sweep. Then, AuNPs (Au nanoparticles) were electroplated on the Au electrode with a constant $V=-0.2 \mathrm{~V}$ sweep for $200 \mathrm{~s}$ in a $0.25-\mathrm{mM}$ chloroauric acid solution. Subsequently, the Au electrode was incubated in $10 \mu \mathrm{g} / \mathrm{mL}$ anti-BNP in a $37^{\circ} \mathrm{C}$ incubator for $3 \mathrm{~h}$. The Au electrode was rinsed with PBST solution (PBS:Tween-20 =1000:1) to remove the excess anti-BNP. BSA solution ( $5 \mathrm{mg} / \mathrm{mL}$ in PBS) was used to block sites on the $\mathrm{Au}$ electrode for $30 \mathrm{~min}$ in a $37^{\circ} \mathrm{C}$ incubator and the electrode was again cleaned with PBST. Finally, the Au electrode was incubated in solutions with different concentrations of BNP at $37^{\circ} \mathrm{C}$ for $1.5 \mathrm{~h}$. EIS (electrochemical impedance spectroscopy) was measured in a mixed solution containing $2 \mathrm{mM}$ potassium ferricyanide, $2 \mathrm{mM}$ potassium ferrocyanide and $0.1 \mathrm{M} \mathrm{KCl}$ after each functionalization step.

For Device A, the functionalization steps before BNP binding were exactly the same as those of the Au electrode. The $\mathrm{Ag} / \mathrm{AgCl}$ reference electrode was biased at $0 \mathrm{~V}$. A constant voltage of $0.4 \mathrm{~V}, V_{\mathrm{DS}}$, was applied between $\mathrm{H}$. F and L. F, and the drain-to-source current $I_{\mathrm{DS}}$ was measured with a Keithley (USA) 2602B SMUA. The voltage $V_{\text {DS-S }}$ between H. S and L. S was measured with SMUB. $I_{\mathrm{DS}}$ and $V_{\text {DS-S }}$ were sampled simultaneously every $500 \mathrm{~ms}$. Traditionally, $I_{\mathrm{DS}}$ is used as the output signal to monitor the change in BNP concentration in the solution. For the Kelvin connection technique, $R_{\mathrm{DS}-4 \text { probe }}=V_{\mathrm{DS}-\mathrm{S}} / I_{\mathrm{DS}}$ was monitored as the output signal. Since $V_{\mathrm{DS}}$ is a constant, $I_{\mathrm{DS}}$ is equivalent to $R_{\mathrm{DS}-2 \text { probe }}=V_{\mathrm{DS}} / I_{\mathrm{DS}}$. Theoretically, $R_{\mathrm{DS}-4 \text { probe }}-R_{\mathrm{DS} \text {-2probe }}$ should be a constant $R_{\mathrm{S}}$, which is the series resistance in the circuit. Different concentrations of BNP (diluted in $0.01 \times \mathrm{PBS}$ solution) were spiked onto the FET gate area, and the responses of the $R_{\mathrm{DS}-4}$ probe and $R_{\mathrm{DS}-2}$ probe were monitored.

For Device B, $10 \mu \mathrm{L}$ magnetic beads $(30 \mathrm{mg} / \mathrm{mL})$ were mixed with $10 \mu \mathrm{L}$ anti-BNP $(0.5 \mathrm{mg} / \mathrm{mL})$ and incubated for $6 \mathrm{~h}$ at $37^{\circ} \mathrm{C}$. Then, MBs were collected with a permanent magnet, and the supernatant was removed. The magnetic beads were washed 3 times with PBS, and then the binding sites were blocked by immersion in $1 \mathrm{mg} /$ $\mathrm{mL}$ BSA solution for $0.5 \mathrm{~h}$. Finally, magnetic beads with a concentration of $5 \mathrm{mg} / \mathrm{mL}$ were added to the surface of Device B and held with an N52 permanent magnet on the back side of the chip. The reason that we did not use the electromagnet was to avoid heat dissipation by the electromagnet, which may influence the temperature of the sensor. In the baseline test with different quiescent working points, the reference electrode was biased from $0 \mathrm{~V}$ to $-3.8 \mathrm{~V}(0 \mathrm{~V},-2 \mathrm{~V},-3 \mathrm{~V},-3.5 \mathrm{~V},-3.8 \mathrm{~V})$, and the $R_{\mathrm{DS}-4 \text { probe }}$ and the gate leakage current $I_{\mathrm{G}}$ were both monitored versus time. In the BNP measurement experiment, the change in $R_{\mathrm{DS}-4 \text { probe }}$ was monitored when different concentrations of BNP were spiked on the open gate area.
All experiments for the 3 types of transducers were repeated at least 3 times to ensure that the results were consistent.

\section{Reagents and materials}

The Au-electrode $\mathrm{CHI} 101$ was purchased from $\mathrm{CH}$ Instruments Ins. Anti-BNP (ab20984) and BNP (ab87200) were purchased from Abcam. Magnetic beads (Dynabeads $^{\mathrm{TM}}$ M-280 Tosylactivated) were purchased from Thermo Fisher Scientific.

\section{Acknowledgements}

This work was supported in part by the National Natural Science Foundation of China under Grants 52077200 and 51807175 ,and in part by the Zhejiang Natural Science Outstanding Young Scholar Foundation under Grant LR21E070001.

\section{Author details}

${ }^{1}$ College of Electrical Engineering, Zhejiang University, 310027 Hangzhou, China. ${ }^{2}$ Biosensor National Special Laboratory, Department of Biomedical Engineering, Zhejiang University, 310027 Hangzhou, China. ${ }^{3}$ Hangzhou Global Scientific and Technological Innovation Center, Zhejiang University, 310027 Hangzhou, China

\section{Author contributions}

Experiment, analysis, writing by H.Z.; experiments by Y.G.; conception of the idea, review and editing by S.Y.; review and editing by K.S.; review and editing by P.W. All authors have read and agreed to the published version of the manuscript.

Conflict of interest

The authors declare no competing interests.

Supplementary information The online version contains supplementary material available at https://doi.org/10.1038/s41378-021-00278-7.

Received: 18 December 2020 Revised: 16 March 2021 Accepted: 26 April 2021

Published online: 01 July 2021

\section{References}

1. Szunerits, S., Mishyn, V., Grabowska, I. \& Boukherroub, R. Electrochemical cardiovascular platforms: current state of the art and beyond. Biosens. Bioelectron. 131, 287-298 (2019).

2. Alawieh, H., El Chemaly, T., Alam, S. \& Khraiche, M. Towards point-of-care heart failure diagnostic platforms: BNP and NT-proBNP biosensors. Sensors 19, 5003 (2019).

3. Lu, X. et al. BNP as a marker for early prediction of anthracycline-induced cardiotoxicity in patients with breast cancer. Oncol. Lett. 18, 4992-5001 (2019).

4. Woo, K., Kang, W., Lee, K., Lee, P. \& Lee, H. H. Enhancement of cortisol measurement sensitivity by laser illumination for AlGaN/GaN transistor biosensor. Biosens. Bioelectron. 159, 112186 (2020).

5. Sarangadharan, I. et al. High sensitivity cardiac troponin I detection in physiological environment using AlGaN/GaN High Electron Mobility Transistor (HEMT) Biosensors. Biosens. Bioelectron. 100, 282-289 (2017).

6. Sinha, A. et al. An integrated microfluidic system with field-effect-transistor sensor arrays for detecting multiple cardiovascular biomarkers from clinical samples. Biosens. Bioelectron. 129, 155-163 (2019).

7. Bhattacharyya, I. M. et al. Specific and label-free immunosensing of proteinprotein interactions with silicon-based immunoFETs. Biosens. Bioelectron. 132 143-161 (2019).

8. Wang, J., Gu, Z., Miao, B., Zhao, L. \& Li, J. Detection of multiple samples based on AIGaN/GaN high electron mobility transistors and magnetic microbeads. Electroanalysis 31, 2404-2409 (2019). 
9. Li, J-d et al. Detection of prostate-specific antigen with biomolecule-gated AlGaN/GaN high electron mobility transistors. J. Micromech. Microeng. 24, 075023 (2014).

10. Sarangadharan, I., Huang, S.-W., Kuo, W.-C., Chen, P.-H. \& Wang, Y.-L. Rapid detection of NT-proBNP from whole blood using FET based biosensors for homecare. Sens. Actuat. B Chem. 285, 209-215 (2019).

11. Zhang, H., Tu, J., Yang, S., Sheng, K. \& Wang, P. Optimization of gate geometry towards high-sensitivity AlGaN/GaN pH sensor. Talanta 205, 120134 (2019).

12. Dai, Y. et al. Analysis and experiment of the sensitivity of $A l G a N / G a N$ based heterostructure all-solid-state pH sensor. AIP Adv. 9, 095066 (2019).

13. Dong, $Y$. et al. AlGaN/GaN heterostructure $\mathrm{pH}$ sensor with multi-sensing segments. Sens. Actuat. B Chem. 260, 134-139 (2018).

14. Wang, L., Li, L., Zhang, T., Liu, X. \& Ao, J.-P. Enhanced pH sensitivity of AlGaN/ GaN ion-sensitive field effect transistor with $\mathrm{Al} 2 \mathrm{O} 3$ synthesized by atomic layer deposition. Appl. Surf. Sci. 427, 1199-1202 (2018).

15. Xue, D. et al. Enhancing the sensitivity of the reference electrode free AIGaN/ GaN HEMT based $\mathrm{pH}$ sensors by controlling the threshold voltage. Sens. Actuat. B Chem. 306, 127609 (2019).

16. Lee, C.-T. \& Chiu, Y.-S. Gate-recessed AlGaN/GaN ISFET urea biosensor fabricated by photoelectrochemical method. IEEE Sens. J. 16, 1518-1523 (2016).

17. Dong, $Y$. et al. High sensitive $\mathrm{pH}$ sensor based on AllnN/GaN heterostructure transistor. Sensors 18, 1314 (2018).

18. Xue, D. et al. Improved performance of AIGaN/GaN HEMT based $\mathrm{H}^{+}$sensors by surface hydroxylation treatment. Mater. Sci. Semicond. Process 121, 105386 (2021).

19. Encabo, A. B., Howgate, J., Stutzmann, M., Eickhoff, M. \& Sánchez-García, M. Ultrathin GaN/AIN/GaN solution-gate field effect transistor with enhanced resolution at low source-gate voltage. Sens. Actuat. B Chem. 142, 304-307 (2009).

20. Milgrew, M. J., Riehle, M. O. \& Cumming, D. R. in 2008 IEEE International SolidState Circuits Conference-Digest of Technical Papers. 590-638 (IEEE, 2008).

21. Jimenez-Jorquera, C., Orozco, J. \& Baldi, A. ISFET based microsensors for environmental monitoring. Sensors 10, 61-83 (2010).

22. Bergveld, $P$. Thirty years of ISFETOLOGY: What happened in the past 30 years and what may happen in the next 30 years. Sens. Actuat. B Chem. $\mathbf{8 8}, 1-20$ (2003).

23. Van Hal, R., Bergveld, P., Engbersen, J. F. \& Reinhoudt, D. Characterization and testing of polymer-oxide adhesion to improve the packaging reliability of ISFETs. Sens. Actuat. B Chem. 23, 17-26 (1995).

24. Hammond, P. Encapsulation of a liquid-sensing microchip using SU-8 photoresist. Microelectron. Eng. 73-74, 893-897 (2004).

25. Oelßner, W. et al. Encapsulation of ISFET sensor chips. Sens. Actuat. B Chem. 105, 104-117 (2005).
26. Wu, A., Wang, L., Jensen, E., Mathies, R. \& Boser, B. Modular integration of electronics and microfluidic systems using flexible printed circuit boards. Lab Chip 10, 519-521 (2010).

27. Chovelon, J., Jaffrezic-Renault, N., Cros, Y., Fombon, J. \& Pedone, D. Monitoring of ISFET encapsulation aging by impedance measurements. Sens. Actuat. $B$ Chem. 3, 43-50 (1991).

28. Zhang, H., Yang, S. \& Sheng, K. The safe operating area of AlGaN/GaN based sensor. IEEE Sens. J. 21, 1 (2020).

29. Kokawa, T., Sato, T., Hasegawa, H. \& Hashizume, T. Liquid-phase sensors using open-gate Al Ga N/ Ga N high electron mobility transistor structure. J. Vac. Sci. Technol. B 24, 1972-1976 (2006).

30. Brazzini, T., Bengoechea-Encabo, A., Sánchez-García, M. A. \& Calle, F. Investigation of AllnN barrier ISFET structures with GaN capping for pH detection. Sens. Actuat. B Chem. 176, 704-707 (2013).

31. Zhang, H., Yang, S. \& Sheng, K. The leakage mechanism of the package of the AlGaN/GaN liquid sensor. Materials 13, 1903 (2020).

32. Müntze, G. M. et al. Quantitative analysis of immobilized penicillinase using enzyme-modified AlGaN/GaN field-effect transistors. Biosens. Bioelectron. 64 605-610 (2015).

33. Serafín, V. et al. An electrochemical immunosensor for brain natriuretic peptide prepared with screen-printed carbon electrodes nanostructured with gold nanoparticles grafted through aryl diazonium salt chemistry. Talanta 179 131-138 (2018)

34. Matsuura, $\mathrm{H}$, Sato, $\mathrm{Y}$, Niwa, $\mathrm{O}$. \& Mizutani, F. Electrochemical enzyme immunoassay of a peptide hormone at picomolar levels. Anal. Chem. 77, 4235-4240 (2005)

35. Tai, T.-Y. et al. Design and demonstration of tunable amplified sensitivity of AlGaN/GaN high electron mobility transistor (HEMT)-based biosensors in human serum. Anal. Chem. 91, 5953-5960 (2019).

36. Chen, $Y$. et al. Field-effect transistor biosensor for rapid detection of Ebola antigen. Sci. Rep. 7, 1-8 (2017).

37. Lin, Y.-H. et al. Bottom-up assembly of silicon nanowire conductometric sensors for the detection of apolipoprotein A1, a biomarker for bladder cancer. Microchim. Acta 184, 2419-2428 (2017)

38. Huang, C.-C. et al. AlGaN/GaN high electron mobility transistors for protein-peptide binding affinity study. Biosens. Bioelectron. 41, 717-722 (2013)

39. Wang, Y.-L. et al. Long-term stability study of botulinum toxin detection with AlGaN/GaN high electron mobility transistor based sensors. Sens. Actuat. B Chem. 146, 349-352 (2010).

40. Chu, C.-H. et al. Beyond the Debye length in high ionic strength solution: direct protein detection with field-effect transistors (FETs) in human serum. Sci. Rep. 7, 1-15 (2017). 